\title{
ESTUDIO EXPERIMENTAL DE LAS FRECUENCIAS FUNDAMENTALES DE MODELOS ESTRUCTURALES ANTE SISMOS ESCALADOS Y ARTIFICIALES.
}

\section{Experimental study of the fundamental frequencies of structural models in the face of scaled and artificial earthquakes.}

\author{
Villalba Nieto Paola Ximena* (iD, Guaygua Quillupangui Byron Armando iD, Cepeda Aveiga Ángel \\ Israel iD, Hipocuro Simbaña Narcisa Alexandra (iD.
}

Universidad Central del Ecuador, Facultad de Ingeniería, Ciencias Físicas y Matemática, Quito, Ecuador.

*pxvillalba@uce.edu.ec

$\mathrm{R}$ esumen

La instrumentación de edificaciones nos permite evaluar y analizar el diseño de una edificación existente, e identificar los posibles daños después de la ocurrencia de un evento sísmico moderado a severo, considerando la frecuencia fundamental como parámetro esencial en la Dinámica de Estructuras. El presente estudio tiene como propósito analizar las frecuencias fundamentales de seis modelos estructurales, y comparar los resultados obtenidos con los métodos de análisis descriptivos Modal Espectral y Paso a paso en el tiempo. El análisis experimental se realizó en la mesa de vibración Quanser XY Shake Table III; empleando tres registros sísmicos corticales y tres por subducción, además de cuatro acelerogramas artificiales generados apropiadamente. La fase experimental consistió en la construcción y ensayo de los modelos, encontrando las aceleraciones registradas de los sismos y acelerogramas seleccionados escalados a un desplazamiento de $2.5 \mathrm{~cm}$. Finalmente las respuestas dinámicas obtenidas experimentalmente, fueron llevadas del dominio del tiempo al dominio de la frecuencia a través de la Transformada Rápida de Fourier, para lo cual se desarrolló una subrutina en MATLAB que incluyó el filtro de Butterworth y el suavizado mediante la función de Konno \& Ohmachi, la cual procesa de manera directa las aceleraciones entregadas por los acelerógrafos.

Palabras clave: frecuencias fundamentales, análisis dinámicos, mesa de vibración, transformada fourier.

A bstract

The instrumentation of buildings allows us to evaluate and analyze the design of an existing building, and identify possible damage after the occurrence of a moderate to severe seismic event, considering the fundamental frequency as an essential parameter in the Dynamics of Structures. The purpose of this study is to analyze the fundamental frequencies of six structural models, and to compare the results obtained with the Modal Spectral and Step by step descriptive methods of analysis over time. The experimental analysis was carried out on the Quanser XY Shake Table III vibration table using three cortical and three subduction seismic recordings, in addition to four appropriately generated artificial accelerograms. The experimental phase consisted of the construction and testing of the models, finding the recorded accelerations of the earthquakes and selected accelerograms scaled to a displacement of $2.5 \mathrm{~cm}$. Finally, the dynamic responses obtained experimentally were taken from the time domain to the frequency domain through the Fast Fourier Transform, for which a subroutine was developed in MATLAB that included the Butterworth filter and smoothing using the Konno \& Ohmachi function, which directly processes the accelerations delivered by the accelerometer.

Keywords: fundamental frequencies, dynamic analysis, vibration table, fourier transform. 


\section{INTRODUCCIÓN}

El Ecuador es un territorio sísmicamente activo que durante su historia ha sido el escenario de varios terremotos destructivos, los cuales han sido generados por tres regímenes tectónicos: Cortical, Subducción Interplaca y Subducción Intraplaca.

A pesar de esto, fue en el código Ecuatoriano de la Construcción CEC 2000 publicado en el 2001, en el que por primera vez se presenta un mapa de zonificación sísmica para Ecuador, el cual dividía al país en 4 zonas sísmicas basadas en estudios de peligro sísmico; actualmente se encuentra en vigencia la Norma Ecuatoriana de la Construcción NEC 2015, la cual añade no solo una caracterización al peligro sísmico, sino que presenta además la zonificación sísmica del territorio, contando con 6 zonas.

De la misma forma en la NEC, dentro de las metodologías de diseño sísmico se exponen dos métodos de análisis dinámico lineal, el Modal Espectral y el Paso a paso en el tiempo.

Estos métodos de análisis descriptivos, son una alternativa para el estudio y comprensión de los efectos de excitaciones sísmicas, ya que nos permiten conocer las respuestas máximas de la estructura; pero al ser estos métodos analíticos es importante estudiar sus resultados mediante ensayos experimentales.

La mayoría de los sismos de gran magnitud Mw $>6$ que se han presentado en el país no tienen registros en acelerogramas, ya que en el año 2009 se creó la RENAC (Red Nacional de Acelerógrafos), mientras que en los últimos años la EPN (Escuela Politécnica Nacional) ha desplegado una red sísmica local en la ciudad de Quito, lo que nos lleva a contar con datos únicamente de los últimos años, de acuerdo con (1).

Se hace necesario el estudio de eventos ocurridos a nivel internacional, seleccionados en función de fuentes similares, y también la generación de acelerogramas artificiales.
La frecuencia fundamental (o periodo fundamental) es un parámetro dinámico primordial en el análisis de estructuras sometidas a excitaciones sísmicas, de ahí que en los últimos años se han venido determinando en estructuras existentes mediante instrumentación de sensores sísmicos, utilizando vibraciones forzadas, grabación de sismos y vibraciones ambientales, los cuales a través de la Transformada rápida de Fourier nos permiten pasar del Análisis del dominio del tiempo, al dominio de la frecuencia.

En el estudio realizado por (2), se analizó estadísticamente 152 edificaciones de hormigón armado en la ciudad de Quito, para establecer la variación del periodo fundamental a través de vibraciones ambientales.

\section{MATERIALES Y MÉTODOS}

La etapa experimental consideró la construcción de seis modelos estructurales con materiales encontrados en el mercado.

En la Tabla 1 se muestran las características generales de cada modelo, con una descripción de los materiales usados.

En los modelos de cuatro y seis pisos se acoplaron dos acelerómetros biaxiales, mientras que en los modelos de dos y tres pisos uno. En la Fig. 1 como ejemplo se observa el modelo 2 acoplado en la mesa de vibración con la instalación de dos acelerómetros.

\begin{tabular}{|c|c|c|c|l|}
\hline Modelo & Altura (m) & Pisos & Peso (kg) & \multicolumn{1}{|c|}{ Materiales } \\
\hline 1 & 1.50 & 4 & 34.50 & $\begin{array}{l}\text { MDF, Tubo cuadrado } \\
\text { hueco, tol, Ángulos de } \\
\text { acero }\end{array}$ \\
\hline 2 & 1.70 & 6 & 6.50 & $\begin{array}{l}\text { Varillas roscadas, Acrí- } \\
\text { lico, tuercas, MDF }\end{array}$ \\
\hline 3 & 1.60 & 4 & 18.80 & $\begin{array}{l}\text { Tol, Varilla cuadrada, } \\
\text { Ángulos de acero }\end{array}$ \\
\hline 4 & 0.50 & 2 & 2.65 & $\begin{array}{l}\text { Tol, varilla roscada, } \\
\text { tuercas, MDF }\end{array}$ \\
\hline 5 & 0.85 & 3 & 3.65 & $\begin{array}{l}\text { Acrílico, varilla rosca- } \\
\text { da, tuercas, MDF }\end{array}$ \\
\hline 6 & 1.00 & 2 & 10.45 & $\begin{array}{l}\text { Tol, varilla cuadrada, } \\
\text { ángulos de acero }\end{array}$ \\
\hline
\end{tabular}

Tabla 1. Características de los modelos estructurales. 


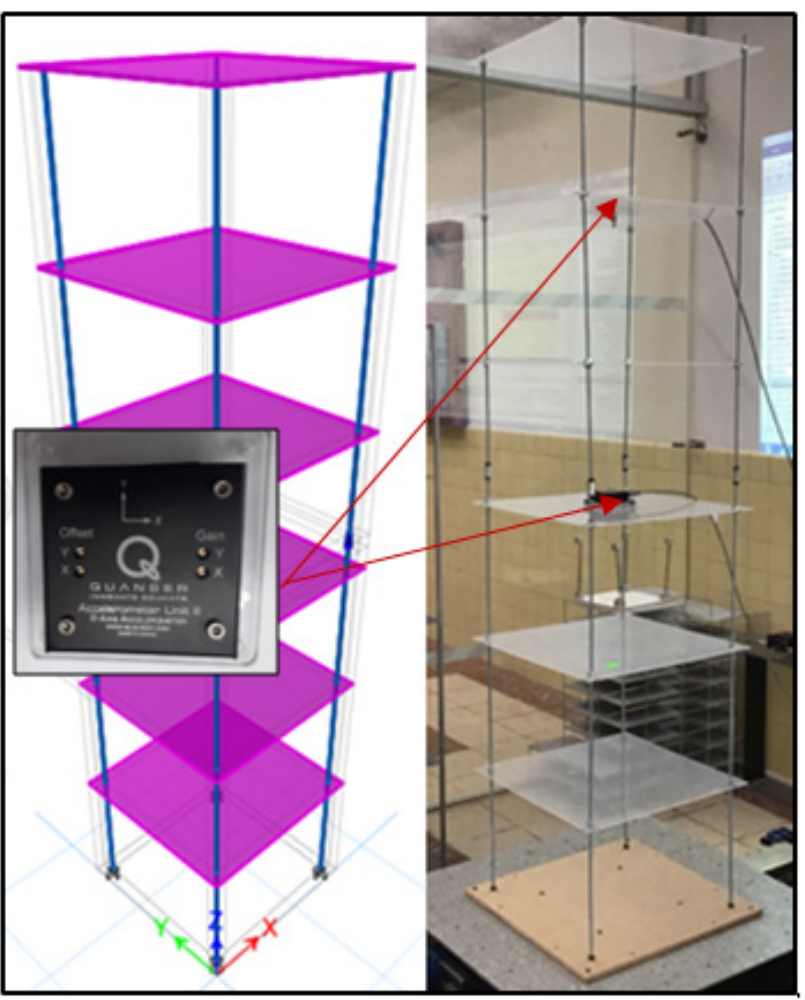

Figura 1. Características de los modelos estructurales. Fuente: Los autores.

Se realizó la comparación de los resultados obtenidos a través de métodos de análisis dinámicos Modal Espectral y Paso a Paso en el Tiempo, con las respuestas obtenidas de los modelos construidos a partir de la aplicación de distintos movimientos sísmicos, simulados a través de la mesa de vibración Quanser XY Shake Table III del Laboratorio de Ensayo de Materiales y Modelos de la Facultad de Ingeniería Ciencias Físicas y Matemática de la Universidad Central del Ecuador.

\section{Registros sísmicos y acelerogramas artificiales utilizados}

Ecuador es un país con alta peligrosidad sísmica, se tiene acceso a datos de registros sísmicos ocurridos en los últimos años, sin embargo, para escenarios críticos se hace necesario la inclusión de registros compatibles de otros países, que a pesar de tener similares características geomorfológicas de terreno, no reflejan la realidad sísmica de la localidad, razón por la cual se utilizó además acelerogramas artificiales.

Se utilizó dos tipos de sismos: subducción e impulsivos, en función a su índice de Impulsividad (Ip) de acuerdo al criterio de (3), que considera sismos impulsivos y fuertemente impulsivos si su índice es menor a 20. Para subducción, los sismos utilizados se encuentran en la Tabla 2 que incluyó el sismo sucedido en Pedernales en abril de 2016, en las estaciones Pedernales APED y Portoviejo APO1.

\begin{tabular}{|c|c|c|c|c|}
\hline Sismo & $\begin{array}{c}\text { Bases de } \\
\text { Datos }\end{array}$ & Magnitud & Estación & Ip \\
\hline $\begin{array}{c}\text { Maule - Chile } \\
(\mathbf{2 0 1 0})\end{array}$ & RENADIC & 8.8 & Angol & 188.32 \\
\cline { 4 - 5 } & RAII- & 8.1 & Llolleo & 118.57 \\
\cline { 4 - 5 } $\begin{array}{c}\text { Michoacán - } \\
\text { México (1985) }\end{array}$ & UNAM & $\begin{array}{c}\text { Caleta de } \\
\text { Campos }\end{array}$ & 35.02 \\
\hline \multirow{2}{*}{$\begin{array}{c}\text { Muisne - Ecua- } \\
\text { dor (2016) }\end{array}$} & RENAC & 7.8 & $\begin{array}{c}\text { Pedernales } \\
\text { APED }\end{array}$ & 79.12 \\
\cline { 4 - 5 } & & $\begin{array}{c}\text { Portoviejo } \\
\text { APO1 }\end{array}$ & 43.24 \\
\hline
\end{tabular}

Tabla 2. Sismos por Subducción

Los registros de los sismos de Quito de agosto de 2014, a pesar de tener un índice de impulsividad mayor a 30, se incluyeron dentro de los sismos impulsivos, debido a que se registraron dentro del DMQ; considerando que en esta ciudad atraviesa un sistema de fallas ciegas. Estos registros fueron escalados a la aceleración máxima del espectro de diseño en aceleraciones establecido en la NEC para un suelo tipo D en la ciudad en mención. Tabla 3.

Para generar los acelerogramas artificiales se utilizó una función transitoria a partir de la metodología propuesta por (3), dicha función es cambiada al dominio de la frecuencia a través de la transformada rápida de Fourier, donde se realiza el ajuste del espectro modificando las amplitudes (4); seguidamente se compara con el espectro objetivo y se cambia de nuevo al dominio del tiempo por medio de la inversa de la transformada rápida de Fourier, para finalmente ajustar la línea base y la aceleración máxima $(6,13-17)$.

\begin{tabular}{|c|c|c|c|c|}
\hline Sismo & $\begin{array}{c}\text { Base de } \\
\text { datos }\end{array}$ & Magnitud & Estación & Ip \\
\hline \multirow{2}{*}{$\begin{array}{l}\text { Northridge - } 01 \text { - } \\
\text { EE.UU. (1994) }\end{array}$} & \multirow{2}{*}{ PEER } & \multirow{2}{*}{6.69} & LA Dam & 10.93 \\
\hline & & & J.F.P.G.B & 14.83 \\
\hline \multirow{2}{*}{$\begin{array}{c}\text { Kocaeli - Turquía } \\
\text { (1999) }\end{array}$} & \multirow{2}{*}{ PEER } & \multirow{2}{*}{7.51} & Duzce & 12.93 \\
\hline & & & Yarimca & 10.56 \\
\hline \multirow{2}{*}{$\begin{array}{c}\text { Quito - Ecuador } \\
(2014)\end{array}$} & \multirow{2}{*}{ RENAC } & 5.1 & $\begin{array}{c}\text { EPN } \\
(12-08-2014)\end{array}$ & 102.94 \\
\hline & & 4.7 & $\begin{array}{c}\text { EPN } \\
(12-08-2014)\end{array}$ & 60.81 \\
\hline
\end{tabular}

Tabla 3. Sismos Impulsivos

Los acelerogramas artificiales consideraron los espectros objetivos de diseño en aceleraciones 
de la NEC tanto en Pedernales como en Quito, y como fuente de intensidad los sismos de Pedernales en la estación de Portoviejo y el del sismo de Quito del 12 de agosto de 2014 respectivamente.

Los registros sísmicos y acelerogramas artificiales fueron escalados a un desplazamiento de $2,5 \mathrm{~cm}$, conservando las aceleraciones originales en las dos direcciones de análisis para la simulación en la mesa de vibración; obteniendo los valores de las aceleraciones en el tiempo en los dos sentidos $\mathrm{x}$ e y de los acelerómetros instalados en cada modelo ensayado. Las aceleraciones registradas por las excitaciones sísmicas tienen un rango de apreciación de $0,0002 \mathrm{~g}$.

\section{Análisis de Fourier}

La Transformada de Fourier es una herramienta matemática capaz de extraer la información frecuencial de una forma de onda una vez conocido su comportamiento temporal y viceversa $(7,18$ 23). Una señal compleja puede ser descompuesta en una serie de armónicos sinusoidales, y expresada mediante la siguiente suma:

$F(t)=\sum_{n=-\infty}^{\infty} a_{n} \cos \left(2 \pi n \omega_{0} t\right)+b_{n} \sin \left(2 \pi n \omega_{0} t\right)$

$F(t)=C_{0} \sum_{n=-\infty}^{\infty} C_{n} \cos \left(n \omega_{0} t-\theta_{n}\right)$

Donde:

$\omega_{0}$ : Es la frecuencia angular fundamental del armónico.

$a_{n}, b_{n}, c_{n}$ y $\theta_{n}$ : Son los coeficientes de Fourier que definen las funciones senoidales múltiplos de la fundamental.

Un filtro es un elemento que discrimina una determinada frecuencia o gama de frecuencias de una señal que pasa a través de él, pudiendo modificar tanto su amplitud como su fase (8). El filtro de Butterworth es muy utilizado para producir la respuesta más plana que sea posible hasta la frecuencia de corte. A medida que aumenta el orden del filtro se incrementan la pendiente de atenuación.

La función de Konno y Ohmachi (9, 24-26), de tipo trigonométrica-logarítmica, considera como frecuencia central, la de mayor amplitud aplicando un coeficiente exponencial (10, 27-29). El control del suavizado se lleva a cabo mediante la aplicación de una constante que varía entre 0 y 100 , es decir entre un suavizado muy fuerte y muy suave, respectivamente. (8), recomiendan este tipo de suavización ya que preserva el número de puntos de alta y baja frecuencia.

Para procesar las señales entregadas por los acelerógrafos utilizados, a fin de llevar a las mismas del dominio del tiempo al dominio de la frecuencia, se desarrolló una subrutina en MATLAB compatible con los datos entregados por el software Quanser, programación propia de la mesa de vibración; que permite tomar los vectores tiempo y aceleración de los Tas medidos por los acelerómetros, separar las frecuencias mayores a $10 \mathrm{~Hz}$ y el ruido de la señal con un filtro pasa-baja de Butterworth, realizar la FFT, suavizar con el método de Konno y Ohmachi y graficar tanto el acelerograma como el espectro de Fourier.

En el espectro de Fourier los picos característicos representan las frecuencias de los modos de vibración, siendo el de mayor amplitud el que corresponde a la frecuencia fundamental, es decir al periodo del primer modo de vibración. En la Fig. 2 se observan los diagramas de las aceleraciones entregadas por los acelerómetros de la mesa de vibración, el acelerograma filtrado, Espectro de Fourier y el correspondiente suavizado obtenido de la subrutina en Matlab desarrollada en este trabajo.

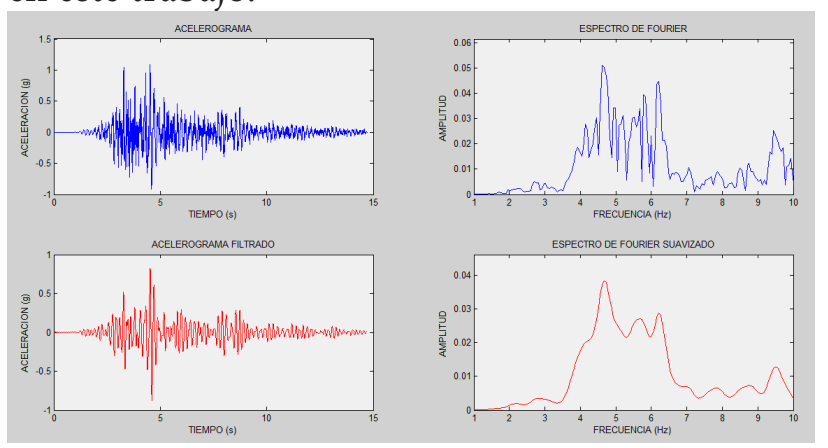

Figura 2. Procesamiento de señales: Espectro de Fourier suavizado Fuente: Los autores.

\section{RESULTADOS}

Se encontró la frecuencia fundamental experimental de los modelos ante la aplicación de los registros sísmicos y acelerogramas artificiales 
escalados, en cada sentido de análisis x e y, dividiendo los resultados tanto para sismos por subducción como impulsivos, encontrando el valor promedio de cada modelo ensayado.

En la Fig. 3 se observan los Espectros suavizados de Fourier de los seis modelos con su promedio para el sentido de análisis $\mathrm{x}$, ante la aplicación de sismos por subducción, indicándose las amplitudes máximas y sus correspondientes frecuencias y periodos. Se puede apreciar que en el modelo 2 la frecuencia fundamental no corresponde aparentemente a la amplitud de mayor valor; esto sucede por la apreciación del acelerómetro uti-
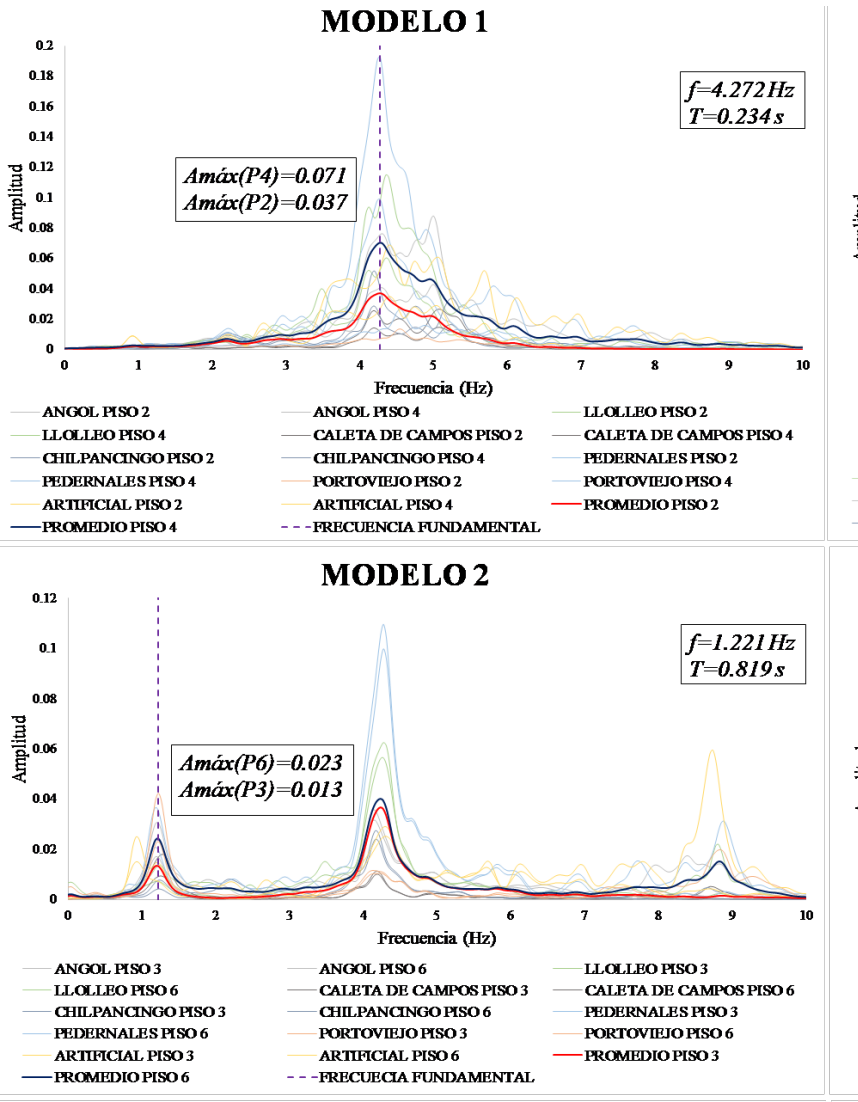

MODELO 3

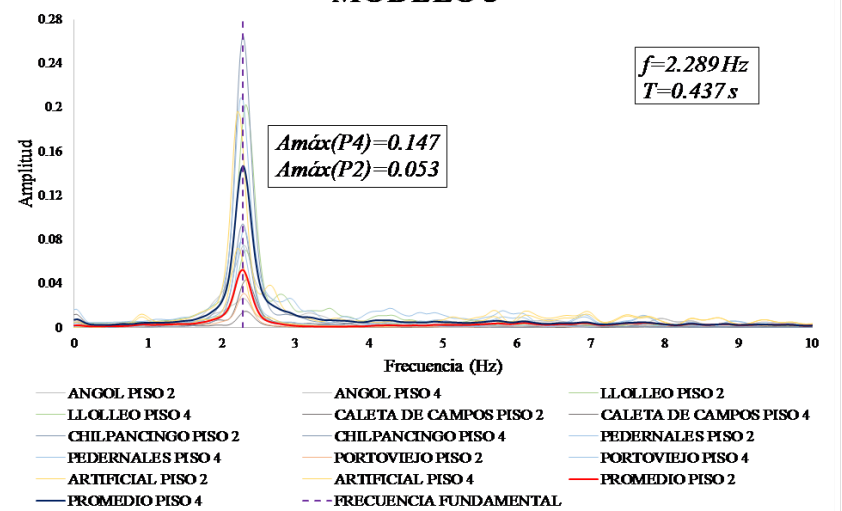

lizado, sin embargo se verificó el valor correcto tanto con la aplicación de sismos impulsivos y los modelos matemáticos realizados. En la Fig. 4 se observan los Espectros suavizados de Fourier de los seis modelos con su promedio para el sentido de análisis $\mathrm{x}$, ante la aplicación de sismos impulsivos.

En la Fig. 5 se muestran los valores de las frecuencias fundamentales de los seis modelos obtenidos de los análisis dinámicos Modal espectral, Paso a paso (Tiempo historia) tanto para los sismos impulsivos como no impulsivos, y los obtenidos experimentalmente.

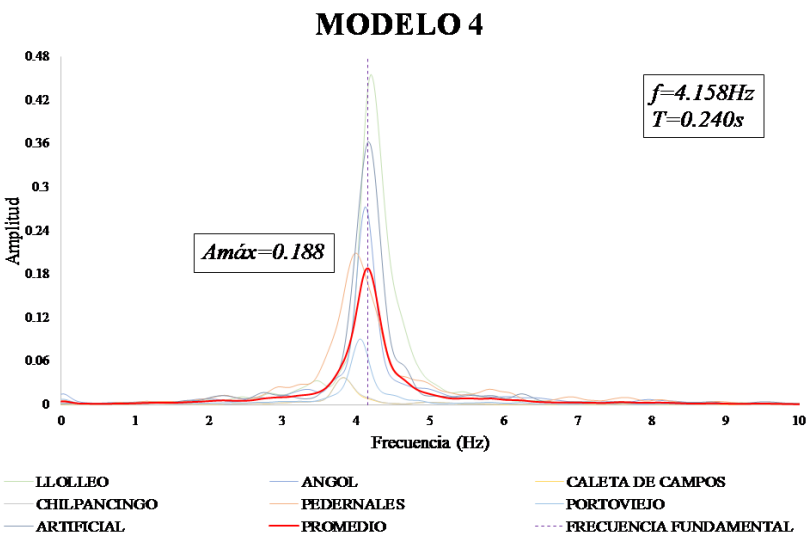

MODELO 5
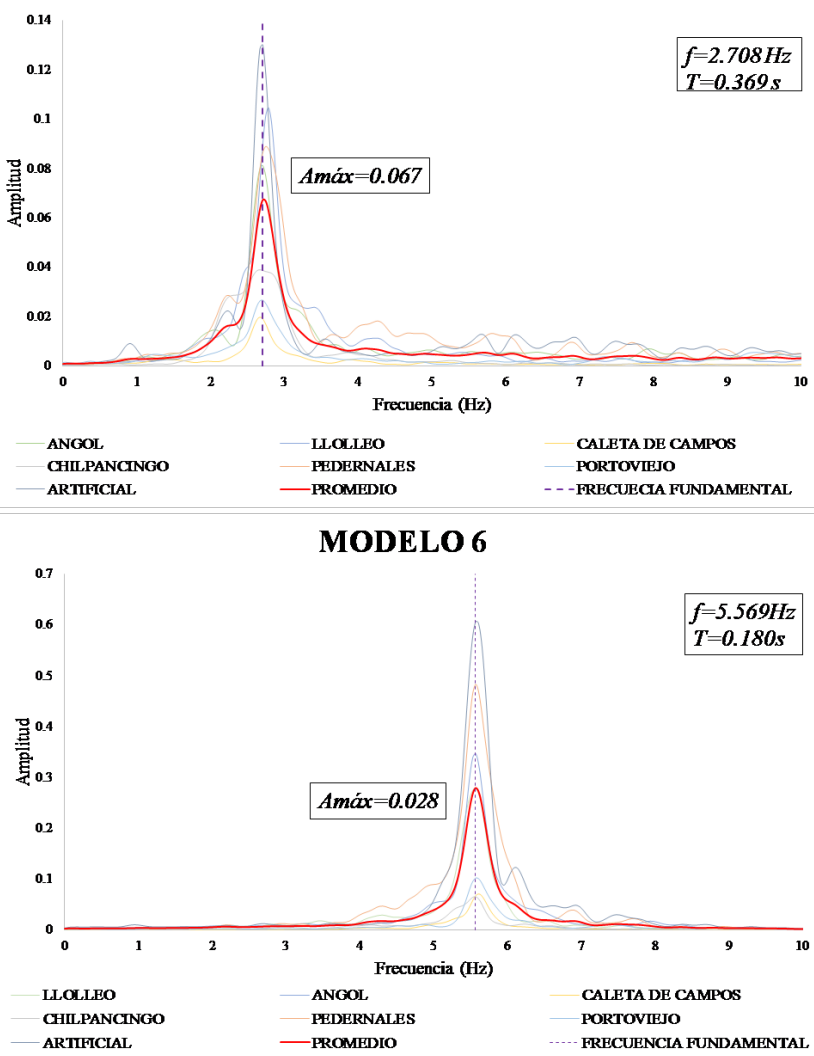

Figura 3. Frecuencias fundamentales de los modelos ante sismos por subducción Fuente: Los autores. 
a) Modelo 1: El porcentaje de variación entre el análisis Modal Espectral y Paso a paso fue de $1.24 \%$, mientras que para los análisis teóricos y experimental de $8.87 \%$. La selección de los materiales fue adecuada, las conexiones viga - columna trabajaron apropiadamente $y$, a pesar de que durante la fase experimental se produjo la rotura de uno de los puntos de suelda en la base, los resultados son consistentes.

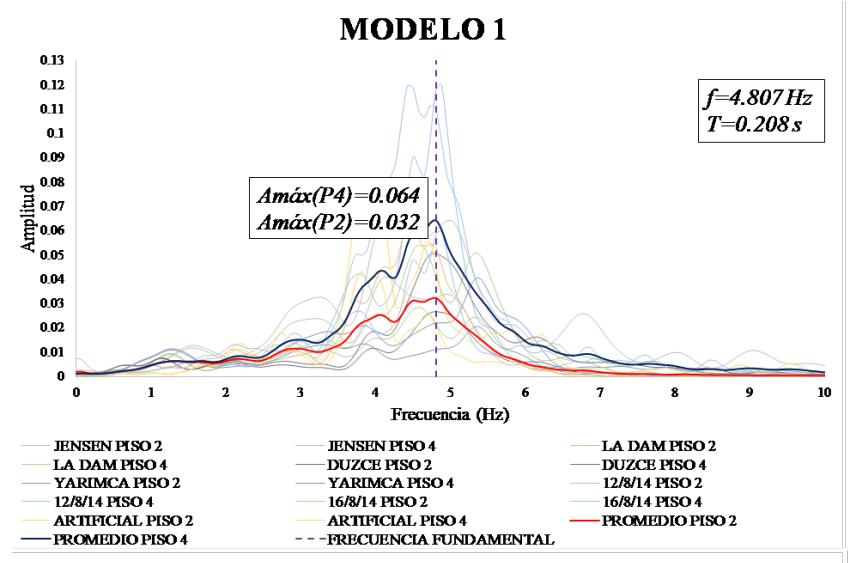

MODELO 2

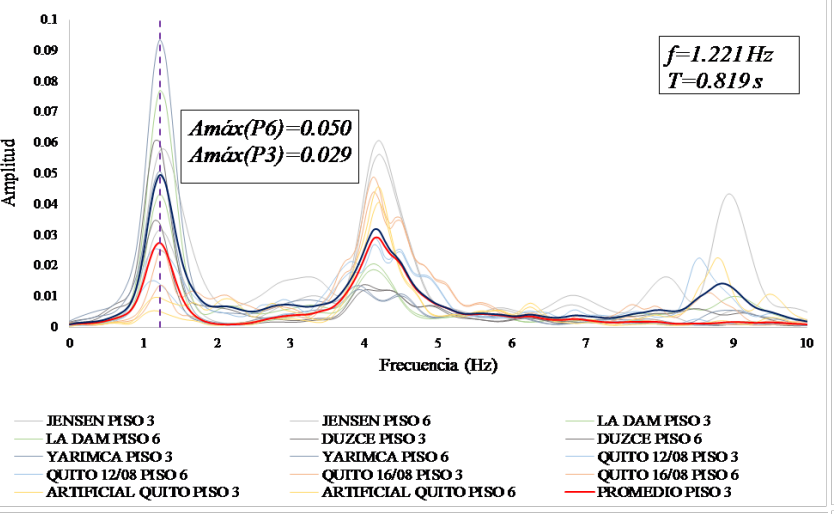

MODELO 3

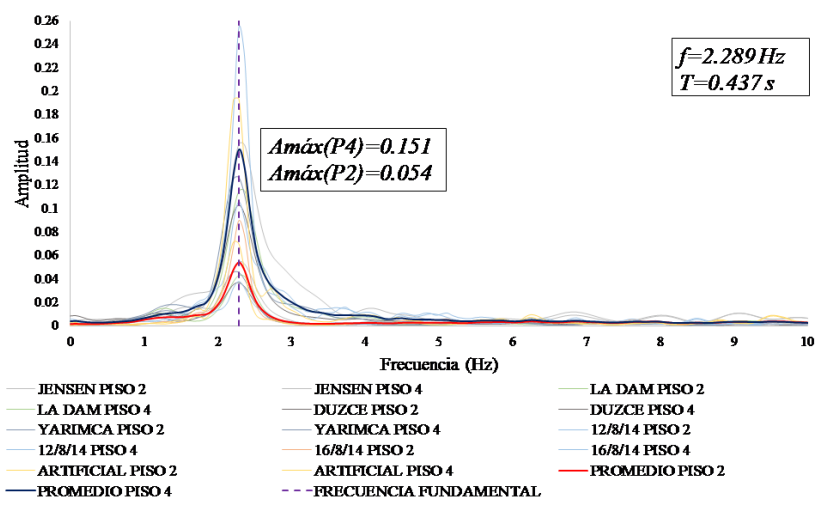

Figura 4. Frecuencias fundamentales de los modelos ante sismos corticales Fuente: Los autores.

c) Modelo 3: el porcentaje de variación entre el análisis Modal Espectral y Paso a paso fue de $0.00 \%$, mientras que para los análisis teóricos y experimental de $4.18 \%$. b) Modelo 2: El porcentaje de variación entre el análisis Modal Espectral y Paso a paso fue de $4.00 \%$, mientras que para los análisis teóricos y experimental de $4.69 \%$.

Al ser de menor frecuencia fundamental, en la fase experimental con sismos por subducción no se logró determinar la frecuencia fundamental en el mayor pico (apreciación de los acelerómetros utilizados).

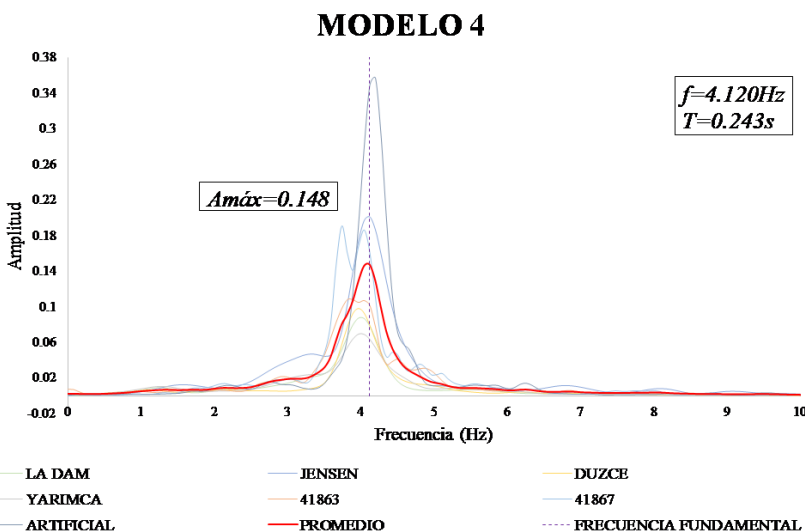

MODELO 5
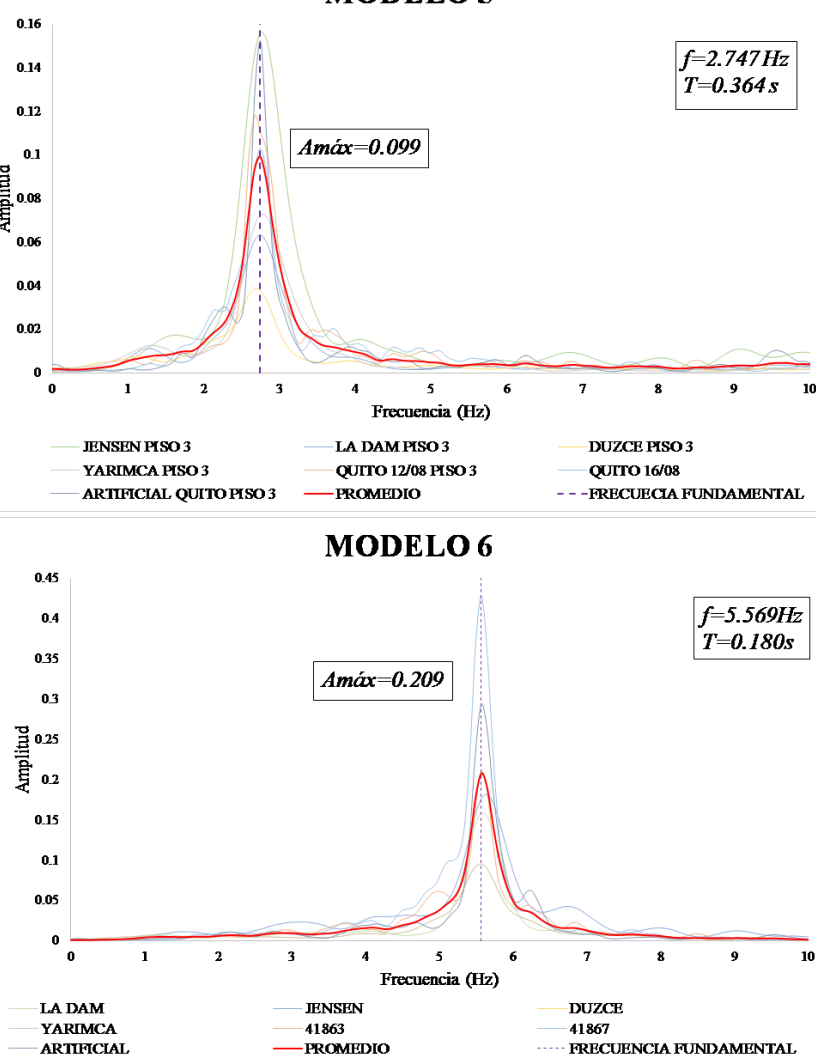

d) Modelo 4: el porcentaje de variación entre el análisis Modal Espectral y Paso a paso fue de $0.20 \%$, mientras que para los análisis teóricos y experimental de $15.87 \%$. 
e) Modelo 5: el porcentaje de variación entre el análisis Modal Espectral y Paso a paso fue de $0.92 \%$, mientras que para los análisis teóricos y experimental de $16.81 \%$. A pesar de contar con materiales similares al modelo 2, al tener una menor altura, la estructura es muy rígida, pudiendo influir las conexiones mediante tuercas en el resultado final.

f) Modelo 6: el porcentaje de variación entre el análisis Modal Espectral y Paso a paso fue de $0.52 \%$, mientras que para los análisis teóricos y experimental de $4.17 \%$, lográndose una excelente simulación de los elementos, conexiones y bases.

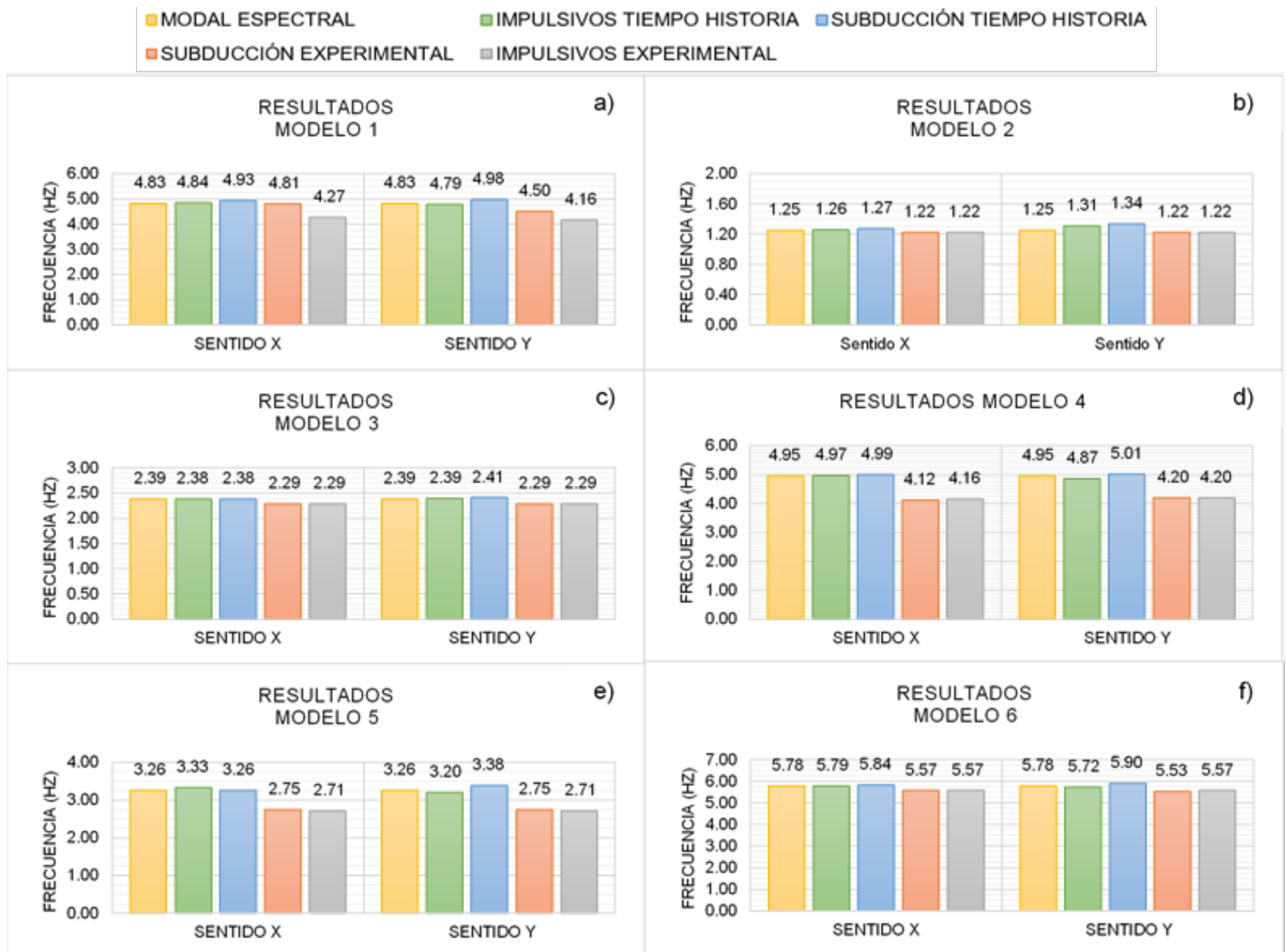

Figura 5. Comparación de los resultados de las frecuencias fundamentales teóricas y experimentales

Fuente: Los autores.

El porcentaje de variación entre el promedio de los valores obtenidos en el análisis Paso a paso y el Modal Espectral fue para el caso de sismos por subducción de 1,93\%, y para sismos impulsivos de $0,75 \%$.

La diferencia en porcentaje entre el promedio de los valores obtenidos en los análisis dinámicos teóricos y los experimentales fue para el caso de sismos por subducción de $10,25 \%$, y para sismos impulsivos de $12,63 \%$.

Si tomamos como referencia el estado del arte en el país, donde los resultados alcanzan una aproximación del $90 \%$ entre valores experimentales y teóricos de respuestas en aceleraciones, de acuerdo con $(11,12)$, los resultados alcanzados en esta investigación son congruentes. La variación de los valores obtenidos teóricos y experimentales es mínima, considerando los múltiples factores que intervienen en la fabricación de los modelos y realización del ensayo.

Hay que indicar que el presente estudio incluye parámetros adicionales, como la fabricación de modelos con múltiples grados de libertad, aplicación de aceleraciones de sismos escalados y artificiales en los dos sentidos de análisis simultáneas, respecto a las consideraciones tomadas en los estudios de referencia mencionados. 


\section{CONCLUSIONES}

El estudio de las frecuencias fundamentales a través de metodologías como la grabación de sismos, vibraciones forzadas o vibraciones ambientales, ha comenzado a tomar relevancia en Ecuador, encontrándose estructuras con instrumentación propia, y requiriéndose tanto en el ámbito público como en el privado la realización de ensayos de vibraciones ambientales, por lo que el estudio experimental de la Dinámica de estructuras se vuelve primordial.

A través de la generación de acelerogramas artificiales, utilizando como fuente de intensidad registros sísmicos reales, se logró obtener acelerogramas compatibles con un sitio específico, los cuales fueron replicados en la mesa de vibración con éxito y que conjuntamente con sismos reales escalados a desplazamientos, se simularon y midieron las aceleraciones registradas en seis modelos construidos con materiales accesibles en el mercado. Para estudiar el dominio de las frecuencias de los resultados entregados por los acelerógrafos en los modelos experimentales, se utilizó la transformada rápida de Fourier, a través del filtro de Butterworth, obteniendo el espectro de frecuencias suavizado mediante la función de Konno \& Ohmachi.

Los valores conseguidos en los modelos experimentales y los obtenidos en los análisis Modal Espectral y Paso a Paso en el Tiempo, tienen porcentajes de variaciones entre $4 \%$ y $17 \%$, siendo el modelo 6 construido con losas de tol, columnas de varillas cuadradas unidas mediante puntos de suelda, y la base mediante ángulos, el que obtuvo los mejores resultados, demostrándose la importancia de considerar simular apropiadamente el empotramiento en la base. La frecuencia fundamental

R eferencias

1. Taipe L. Análisis de las ecuaciones de predicción de movimientos de suelo para el Ecuador utilizando datos registrados durante el periodo 2000 - 2011 en las estaciones sísmicas de banda ancha y acelerógrafos. Escuela Politécnica Nacional. Facultad de Ingeniería Civil y Ambiental. Quito. Ecuador; 2013.

2. Parra K. \& Sarango J. Análisis estadístico del periodo experimental de vibración en edificios aporticados de hormigón armado en el Distrito Metropolitano de Quito. Escuela Politécnica Nacional. Facultad de Ingeniería Civil y Ambiental. Quito. Ecuador; 2016.

3. Panella D. Frau C. Tornello M. IDENTIFICACIÓN DE REGISTROS SÍSMICOS IMPULSIVOS PARA. Mecánica Computacional. 2013.

4. Gasparini, D. A., and Vanmarcke, E. H. Simulated earthquake motions compatible with prescribed response spectra. Evaluation of Seismic Safety of Buildings Report No. 2, Department of Civil Engineering, Massachusetts Institute of Technology, USA; 1976.

5. Marco Mucciarelli, Angelo Masi, Maria Rosaria Gallipoli, Paolo Harabaglia, Analysis of RC Building Dynamic Response and Soil-Building Resonance Based on Data Recorded during a Damaging Earthquake (Molise, Italy, 2002); 2004.

6. Paspuezán P. \& Vargas D. Evaluación de las respuestas máximas obtenidas a través del análisis paso a paso en el tiempo con acelerogramas artificiales generados mediante software, en cuatro edificios de distinto período, ubicados en Quito y Pedernales. Universidad Central del Ecuador. Facultad de Ingeniería. Ciencias Físicas y Matemática. Quito. Ecuador; 2018.

7. Carrillo C. Fundamentos del Análisis de Fourier. Vigo, España; 2003.

8. Sandoval V. \& Vera M. Estudio de la robustez de la estimación espectral de la técnica H/V de Nakamura para Estudios de Caracterización Dinámica de Suelos. Valdivia, Chile: Universidad Austral de Chile. Obtenido de http://cybertesis.uach.cl/tesis/uach/2013/bmfcis218e/doc/bmfcis218e.pdf; 2013.

9. Konno K. \& Ohmachi T. Ground-Motion Characteristics Estimated from Spectral Ratio between Horizontal and Vertical Components of Microtremor. Bulletin of the Seismological Society of America. 228-241. Obtenido de http://www.eq.db.shibaura-it.ac.jp/papers/Konno\&Ohmachi1998.pdf; 1998.

10. Jaramillo A. Piña J. \& Aguilar R. Aplicación del método de cocientes espectrales para la reconfiguración detallada del mapa de Isoperiodosen la cuenca del valle de México. Artículo. Sociedad Mexicana de Ingeniería Geotécnica.A.C. Cancún - México. Obtenido de https://www.smig.org.mx/admArticulos/eventos/1_Reunion_Cancun/2_XXVI_Reunion_Nacional/6_Geotecnia_sismica/I5AGBH_1.pdf; 2012. 
11. Stacey J. Remodelación de mesa de exitación dinámica: Comportamiento dinámico de estructuras a escala. Tesis de pregrado. Universidad San Francisco de Quito. Colegio de Ciencias e Ingeniería. Quito. Ecuador; 2015.

12. Colcha V. \& Tibán E. Estudio comparativo del comportamiento dinámico de modelos estructurales teóricos y modelos estructurales experimentales. Universidad Central del Ecuador. Facultad de Ingeniería. Ciencias Físicas y Matemática. Quito. Ecuador; 2018.

13. Aguiar R. Análisis Sísmico de Edificios. Centro de Investigaciones Científicas. Escuela Politécnica del Ejército; 2008.

14. Aguiar R. Dinámica de Estructuras con CEINCI-LAB. Segunda Edición. Centro de Investigaciones Científicas. Universidad de las Fuerzas Armadas; 2012.

15. Alfonso V. El riesgo sísmico en Quito: análisis y simulaciones. Master 2 SGT Prefalc "Ciencias y Gestión del Territorio". Geología. Riesgos y Gestión del territorio; 2010

16. Architectural Institute of Japan. Earthquake Motion and Ground Conditions in Commemoration of the 20th Anniversary of the Research Subcommitee on Earthquake Ground Motion. the Arquitectural Institute of Japan. Tokio, Japón; 1993.

17. Beauval C, Yepes H, Bakun W, Egred J, Alvarado A, Singaucho J. Locations and magnitudes of historical earthquakes in the Sierra of Ecuador (1587 - 1966). Geophysical Journal International 181. 1613 - 1633; 2010. 18. Carrillo J. Bernal N. \& Porras P Evaluación del diseño de una pequeña mesa vibratoria para ensayos en Ingeniería Sismo-Resistente. Ciencia e Ingeniería Neogranadina. 89-105. Obtenido de http:// www.redalyc.org/comocitar.oa?id=91129721006; 2013.

19. Cepeda A. \& Hipocuro N. Análisis de las frecuencias fundamentales de modelos estructurales con excitaciones sísmicas. Tesis de pregrado. Universidad Central del Ecuador. Facultad de Ingeniería. Ciencias Físicas y Matemática. Quito. Ecuador; 2019.

20. Chango D. Análisis Teórico experimental de la variación del período fundamental durante el proceso de construcción de un edificio de acero. Escuela Politécnica Nacional. Facultad de Ingeniería Civil y Ambiental. Quito. Ecuador; 2016.

21. Dr. Erol Kalkan P. Smoothing function for Fourier Amplitude Spectrum. Obtenido de MATLAB Central File Exchange: https://www.mathworks.com/matlabcentral/fileexchange/70217-smoothing-function-for-fourier-amplitude-spectrum?s_tid=FX_rcl_behav; 2019.

22. Dunand F. Bard P. Y. Chatelain J. Guéguen P. Vassail T. \& Farsi M. Damping and frequency from randomdec method applied to in situ measurements of ambient vibrations. 12th ECEE. ID Paper 869;

23. Espinoza, F. Determinación de Características Dinámicas de Estructuras. Barcelona. Obtenido de https:// www.tdx.cat/handle/10803/6221; 1999.

24. Esquivel L. \& Miranda P. Frecuencia fundamental de entrepisos: Métodos analíticos y validación experimental. Ingeniería. Obtenido de https://revistas.ucr.ac.cr/index.php/ingenieria/article/view/11084/15655; 2013.

25. Flores H. Lomnitz C. \& Yussim S. Estimación de velocidades de fase a partir del análisis de microtremores con método SPAC. México DF. México; 2004

26. González G. Series de Fourier. Transformadas de Fourier y Aplicaciones. Divulgaciones Matemáticas. 4360. Obtenido de http://divmat.demat-fecluz.org/volumenes-anteriores/vol-5-no-1-2-1997; 1997.

27. Ivanovic S. Trifunac M. \& Todorovska M. Ambient vibration tests of structures a review. ISET Journal of Earthquake Technology. 165-197; 2000.

28. James J. F. A Student's Guide to Fourier Transforms with Applications in Physics and Engineering. Cambridge: Cambridge University Press. Obtenido de www.cambridge.org/9780521176835; 2011.

29. Salas P. \& Campos I. Filtros no Lineales. Tlatemoani. Obtenido de http://www.eumed.net/rev/tlatemoani/17/filtros.html; 2014. 